\title{
A novel micromechanical flow controller
}

\author{
M W van Toor $\dagger$, T S J Lammerink $\dagger$, J G E Gardeniers $\dagger$, \\ M Elwenspoek $\dagger \ddagger$ and D Monsma $\dagger$
}

$\dagger$ MESA Research Institute, University of Twente, Department of Electrical

Engineering/Applied Physics, PO Box 217, 7500 AE Enschede, The Netherlands

Presented on 21 October 1996, accepted for publication on 7 April 1997

Abstract. A new concept for a micromechanical flow regulator is presented. Regulation of the flow is achieved using variation of channel length instead of channel diameter. Several design concepts together with their application in fluidic systems are presented. A regulator for biomedical use, as a part of a glaucoma filter implant, has been realized and tested.

\section{Introduction}

The interest in miniaturized fluidic systems, like e.g. total analysis systems ( $\mu \mathrm{TAS})[1,2]$ and mixed circuit boards [3], has been increasing over the past few years. As a result, the development of miniaturized fluid handling devices like micropumps, -valves, -filters and -mixers has been growing rapidly. Many of these microfabricated components are available nowadays for use in fluidic systems. The microvalve is one of the basic components of such systems. This paper describes a new valve concept for regulating the flow resistance to fluids. Different types of flow regulators (continuous as well as discrete) can be realized with this concept. With a slightly altered design, the controller can also be used as a fluid manipulator to switch a fluid flow from one inlet to several outlets or vice versa. It could possibly also function as a fluid plug injector, which is important in the field of (bio)chemical reaction and analysis ( $\mu$ TAS).

Because of the constant diameter of this valve, it is suitable for fluids containing particles, like many biological fluids (blood, eye fluid). The implantable eye fluid flow 'regulator' in a glaucoma filter implant (GFI) is an example of a biomedical application of this valve. A prototype of this valve has already been fabricated and tested-the results are presented here.

\section{The regulator concept}

To regulate a fluid flow a 'fluid resistor' is used, which usually consists of a valve which can vary the diameter of the fluid channel. The relation between the pressure over a channel and the flow through that channel can be described by the following relation [4]:

$$
p=R \varphi_{v}
$$

$\ddagger$ Tel: X-31-53-4893845. Secr.: X-31-53-4892751. Fax: X-31-534893343. where $\varphi_{v}$ represents the volume flow $\left(\mathrm{m}^{3} \mathrm{~s}^{-1}\right), p$ the pressure difference $(\mathrm{Pa})$ over the channel and $R$ the channel resistance $\left(\mathrm{N} \mathrm{m}^{-5} \mathrm{~s}\right)$. For a square channel with sides $2 a(\mathrm{~m})$, the equation describing the channel resistance in the case of fully developed laminar flow is [5]:

$$
R=\frac{1.78 L}{a^{4}} \mu
$$

with $L(\mathrm{~m})$ the length of the channel and $\mu\left(\mathrm{N} \mathrm{s} \mathrm{m}^{-2}\right)$ the fluid's viscosity. From this formula it is clear that there are two methods to vary the resistance:

(1) variation of the channel cross section $(a)$;

(2) variation of the channel length $(L)$.

The first method is well known and descriptions of various valves in macro- and microapplications can be found (e.g. [5]). The second method is hardly used and, as far as we know, has never been reported in micromechanical literature.

The channel diameter variation method (CDV) has a larger resistance value range compared to the channel length variation method (CLV), but the regulation sensitivity (resistance variation due to a valve displacement) is much larger, which makes the resistance control more difficult. Furthermore, the CLV offers the advantage of a constant channel diameter throughout the device, resulting in a reduced chance of obstruction of the device by fluid particles. This is especially important if the regulator is used with biological fluids.

\section{Various regulator designs}

Figure 1 shows a schematic diagram of the regulator concept. The regulator consists of a fluid channel with an inlet and a separated outlet. The basic idea is that the 'length' of the channel can be adjusted by a sliding mechanism depicted in figure 1 . In the slide a second 


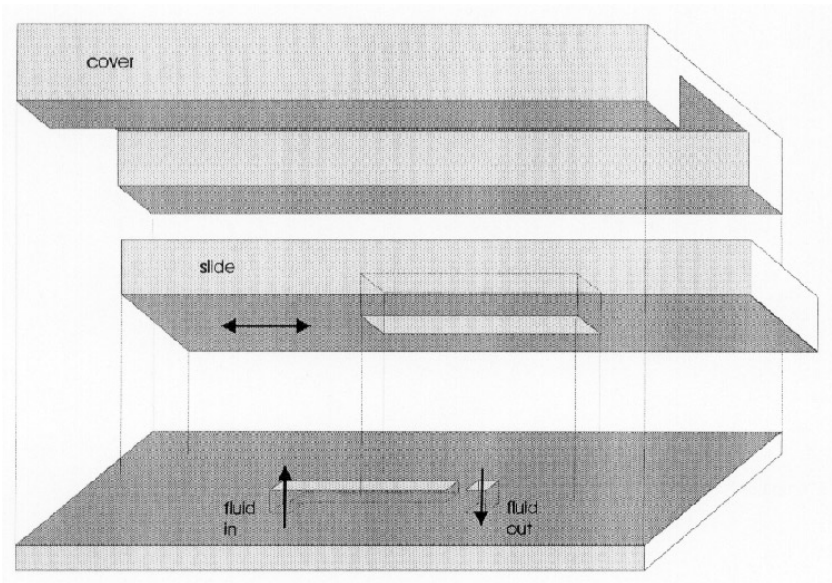

Figure 1. A linear flow resistor.

channel/compartment is present, representing a much lower (zero) resistance than the bottom channel. This resistance can be shunted to the bottom channel at an arbitrary position by the sliding mechanism thus reducing the total resistance of the device. By moving the slide completely to the right (or to the left) the regulator can be closed. The resistance range of the valve is limited and depends on the length of the bottom channel. The range can be increased by using a maze-like channel structure like that shown in figure 2. However, instead of a continuous resistance change a stepwise increase or decrease is obtained in that case.

By covering the complete channel by another layer and introducing holes connecting to the channel at well defined points, the controller can be set accurately at various resistance values. This concept is used in the glaucoma filter implant valve [6], which will be discussed later. The regulator design has been slightly altered for this application (see figure 2).

Using another design the controller can be used as a fluid channel selector/switch. This design has multiple inlets and one outlet (or vice versa) and the slide is designed in such a way that the compartment in the slide is always connected to the outlet, but can only cover one inlet at a time. Of course there can also be a closed position. This type is of importance for $\mu$ TAS systems, where samples and reagents have to be directed to certain places. A model of this valve, with three inlets and one outlet, made of Teflon and powered by a small stepper motor, has successfully been tested. Leakage of fluid under the slide was small, even though the slide surface was not optimized concerning smoothness and fitting to the bottom plate.

Analogons of the regulators mentioned above are presented in figure 3. Figure 3(d) represents a 'fluid plug' injector. This device has two inlets and two outlets and a slide with two compartments (represented by the condensators) which can have two positions. In one position both outlets are connected with their inlets, and the compartments of the slide are filled with the fluid flowing through inlet 1 and 2 respectively. If the slide is now moved to the other position, the compartment filled with fluid 1 connects to inlet and outlet 2, thus 'injecting' fluid 1 into

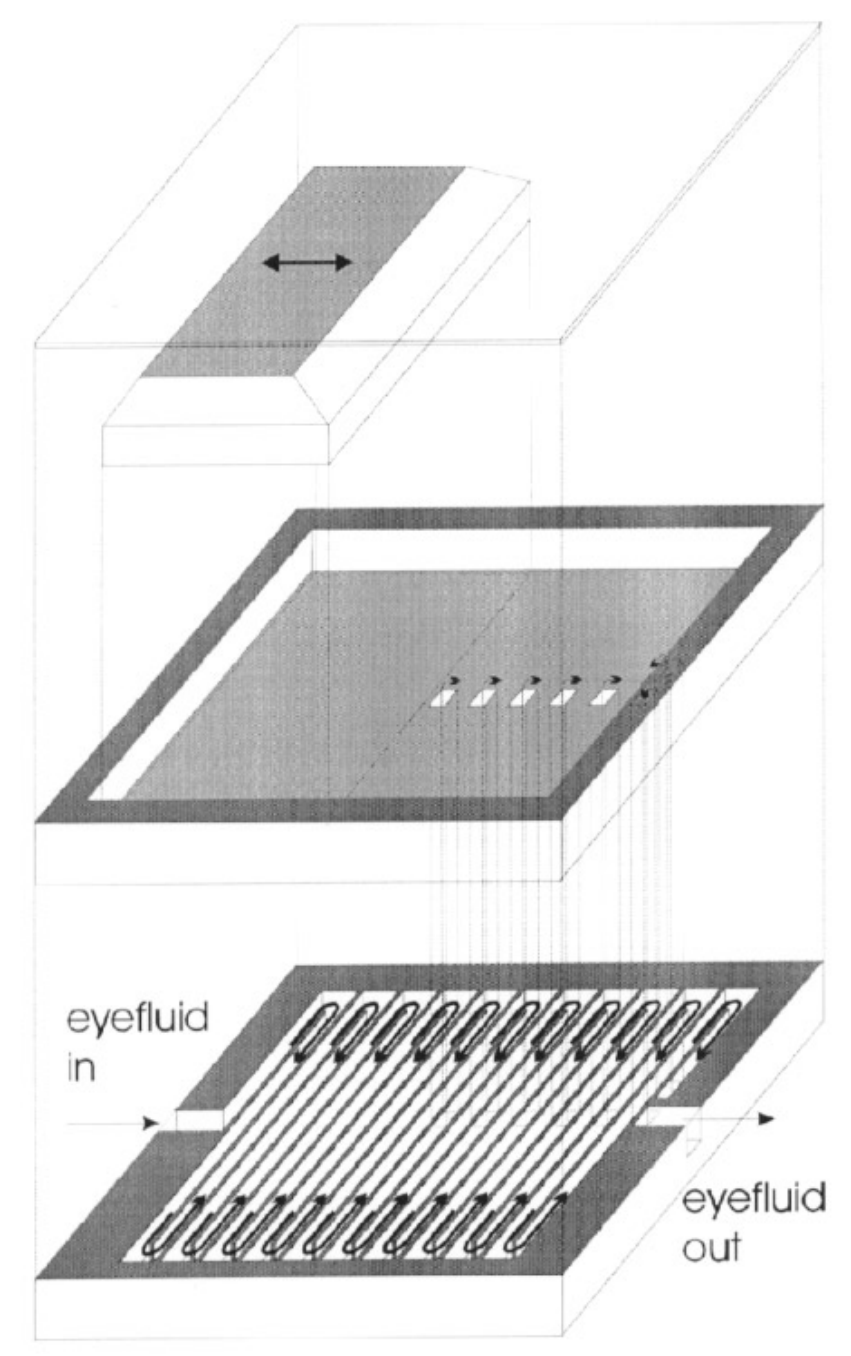

Figure 2. A valve for use in a glaucoma filter implant.

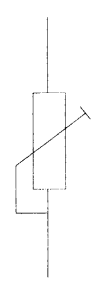

(a)

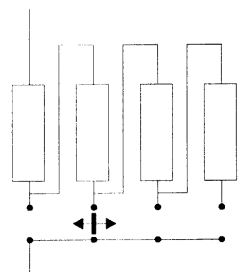

(b)

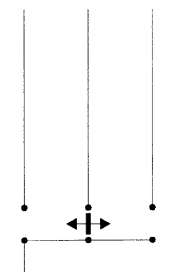

(c)

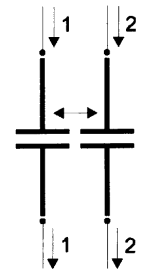

(d)
Figure 3. Analogons (a) continuously adjustable resistor (see figure 1), (b) discreet adjustable resistor (figure 2, GFI valve), (c) three-way switch and (d) selectable volume selector for flow injection analysis.

outlet 2. This kind of device is also of great importance to $\mu$ TAS systems, as a well defined amount of reagent has to be injected for analysis. With this injector the volume of the fluid plug is equal to the volume of the slide compartment.

\section{The glaucoma filter implant valve}

Glaucoma is an eye disease characterized by an elevated intraocular pressure (normally around $15 \mathrm{mmHg}$ 


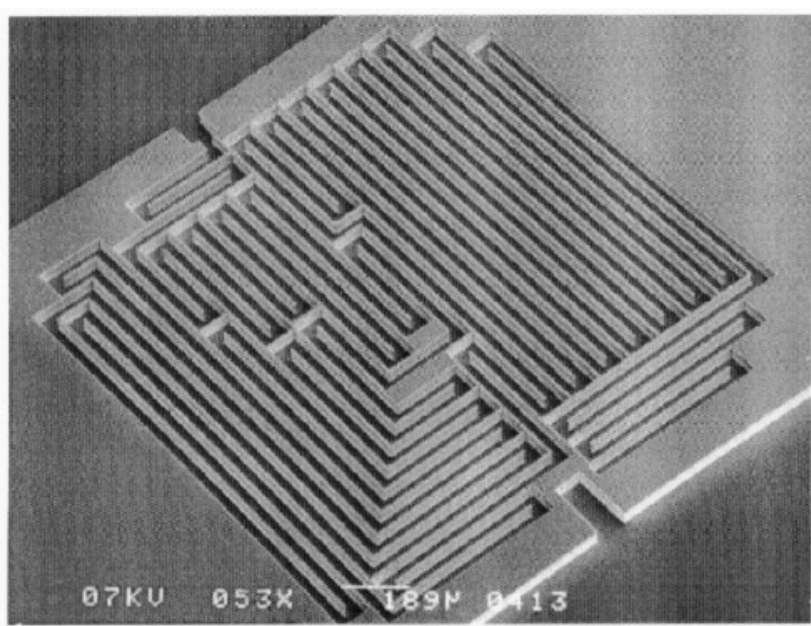

Figure 4. A fluid channel, $50 \times 50 \times 2040 \mu \mathrm{m}^{3}$.

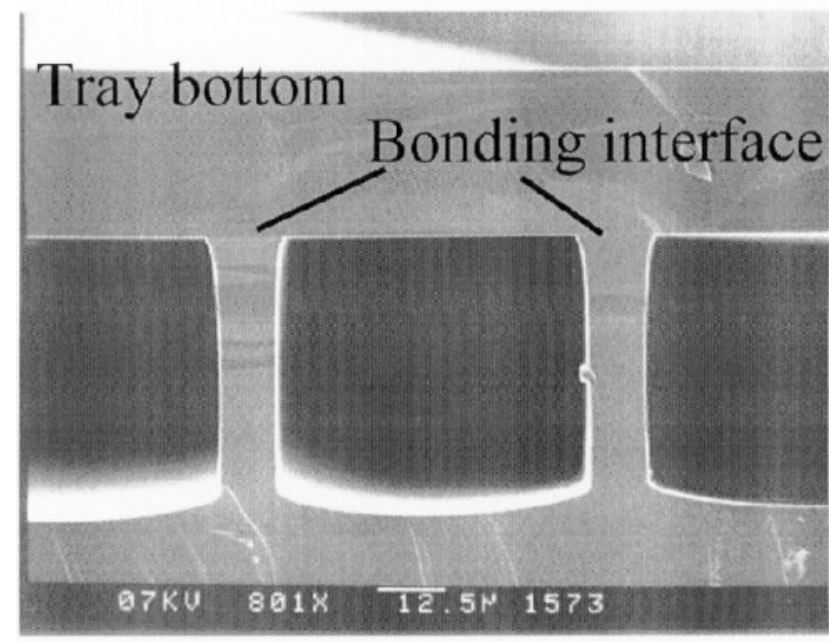

Figure 5. The channel after bonding.

overpressure). Current implants used to relieve this pressure generally consist of a tube, with or without a (one-way) diameter reducing, passive valve, thus providing an alternative pathway for aqueous to leave the eye. The silicon micromachined valve (figure 2), used in conjunction with a GFI, has several advantages over the common implants. It is manually adjustable, giving the surgeon the possibility to change the resistance of the implant even after implantation. This is important to achieve the right intraocular pressure. The valve can also be closed in case of a too low eye pressure. The channel diameter is constant, thus reducing the possibility of obstruction by particles considerably. The different parts of the valve as they have been realized in silicon are depicted in figures 4-6 [6].

The channel of figure 4, which represents the actual resistance to the fluid, has been etched in silicon using RIE with $\mathrm{SF}_{6} / \mathrm{O}_{2}$ plasma. At the top left is the inlet of the channel. The outlet at the bottom right is not directly connected to the channel. This is done to force the fluid through an interconnection to the tray before leaving the valve, thus being able to close the device by moving the slide over the output interconnection (the
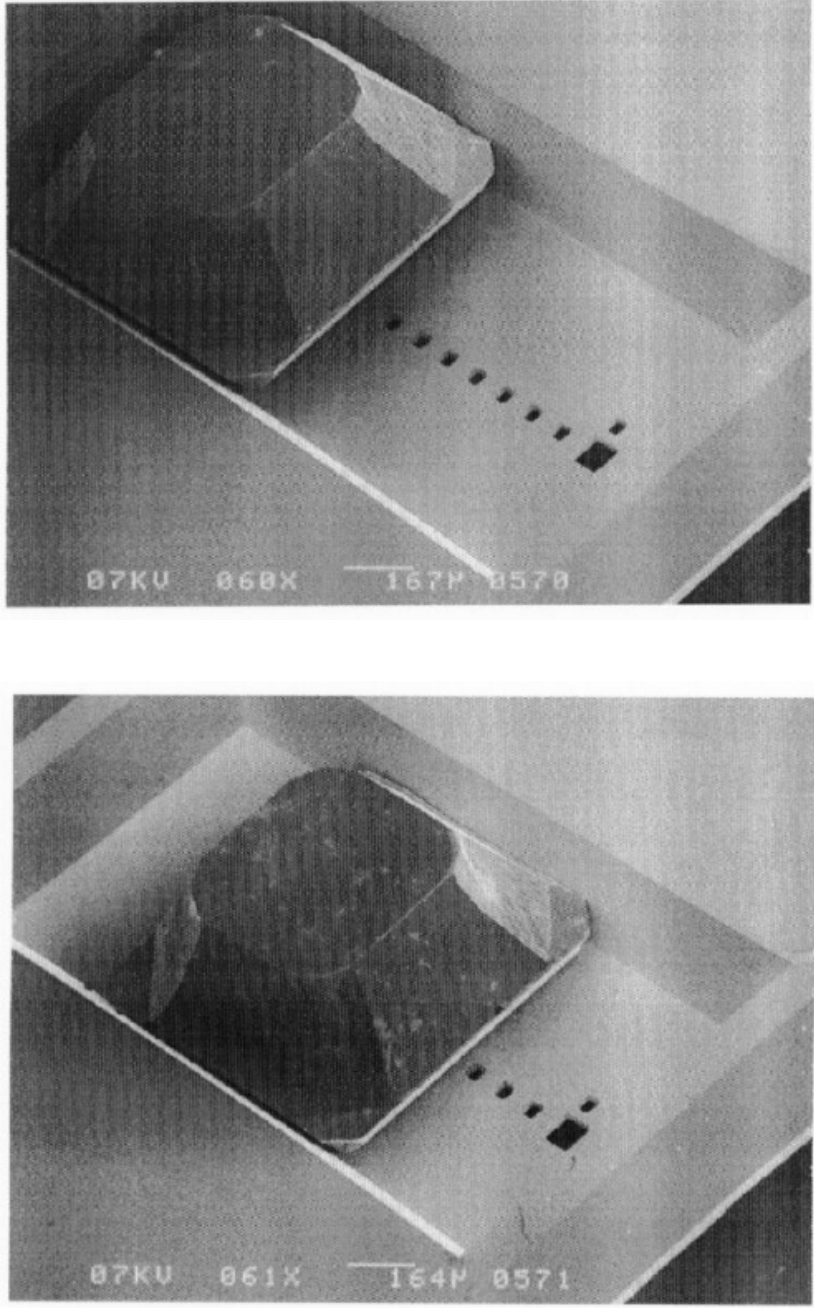

Figure 6. The manually operated sliding mechanism for closing the eight connections.

bigger hole in figure 6). The slide and tray, seen in figure 6 , were both etched with $\mathrm{KOH}$, as this ensures smooth and fitting sidewalls. This is important because the slide has to move inside the tray. The interconnections in the tray, which are $125 \mu \mathrm{m}$ apart, are finally etched from the backside using RIE. There are eight connections which connect to the bottom channel at pre-determined spots, and the bigger hole connects to the outlet. The channel and tray were fusion bonded after an appropriate cleaning step. The bonding resulted in a tight seal with no visible gaps. The resulting channel can be seen in figure 5, which is a cross section through the device after bonding. After bonding, the slide is inserted (figure 6). The valve is finally covered by a Teflon foil held in place by a stainless steel 'lid'. This is shown in figure 7 . Together with silicone rubber gel, applied to the edges of the device before as well as after placement of the foil and stainless steel lid, this ensured a tight leakage-free seal. The Teflon foil also functions to keep the slide in place. By moving the slide manually over the eight connections in the tray bottom with a suitable object (preferably a smooth Teflon tip), eight different resistance values can be chosen. By closing an additional hole by means of 


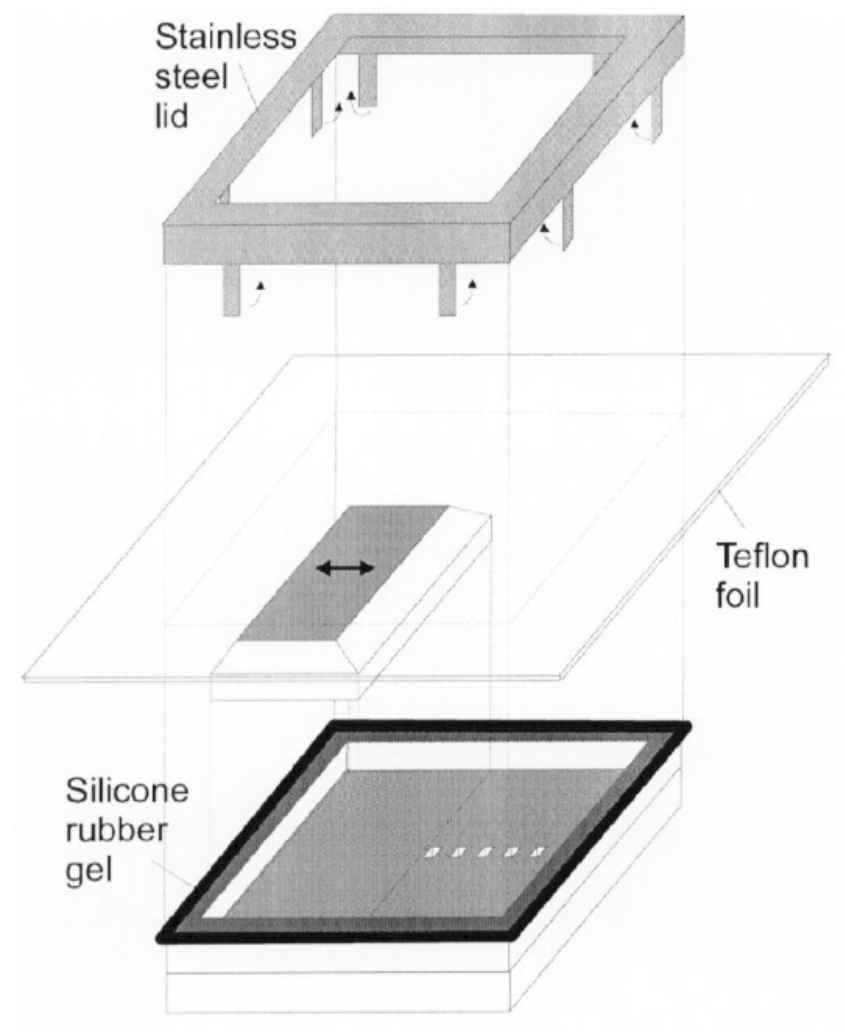

Figure 7. Coverage of the GFI-valve with a Teflon foil clamped by a stainless steel lid.

the slide, the fluid is forced to flow further down the channel until the next open connection is encountered, thus increasing the total resistance of the device. The valve was designed such that the resistance increase is approximately the same for every hole that is additionally closed. The total valve with foil and stainless steel lid is approximately $1500 \times 2500 \times 600 \mu \mathrm{m}^{3}$.

\section{Experimental results}

Pressure flow measurements were performed with four GFI valves using demi-water, which has approximately the same viscosity as eye fluid. The measurement set-up used is shown in figure 8 . A constant volume flow, generated by a syringe pump, was directed through the valve into an open reservoir and the resulting pressure difference between in- and outlet of the valve was measured by a differential pressure transducer. The process of flow generation was controlled by a computer, and data from the pressure sensor was collected by this same computer at a sample rate of $10 \mathrm{~Hz}$. At a constant volume flow the pressure across the valve was measured at various positions of the slide. During the measurements the pressure could be viewed realtime on screen. The slide was moved into another position of different resistance after the pressure had settled to a constant value.

Figure 9 shows a typical measurement result obtained in this way at a volume flow of $2 \mu \mathrm{l} / \mathrm{min}$, which approximately equals the production and removal rate of eye fluid in man. Each time the slide is moved to another

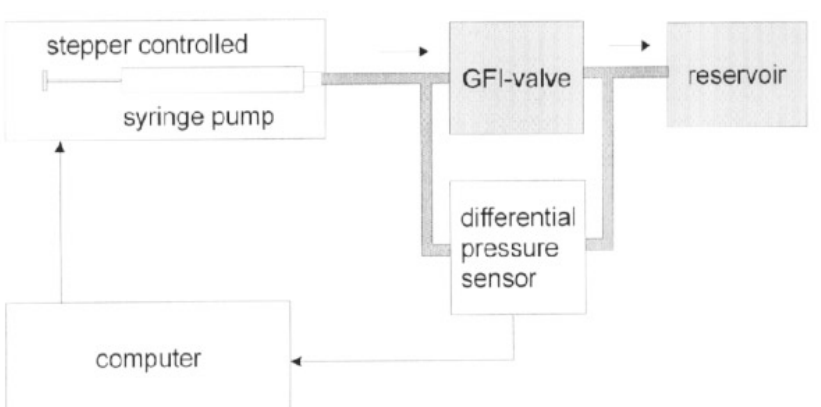

Figure 8. Measurement set-up. The computer-controlled constant flow is forced through the GFI valve into a reservoir and the pressure drop is measured by a differential pressure sensor.

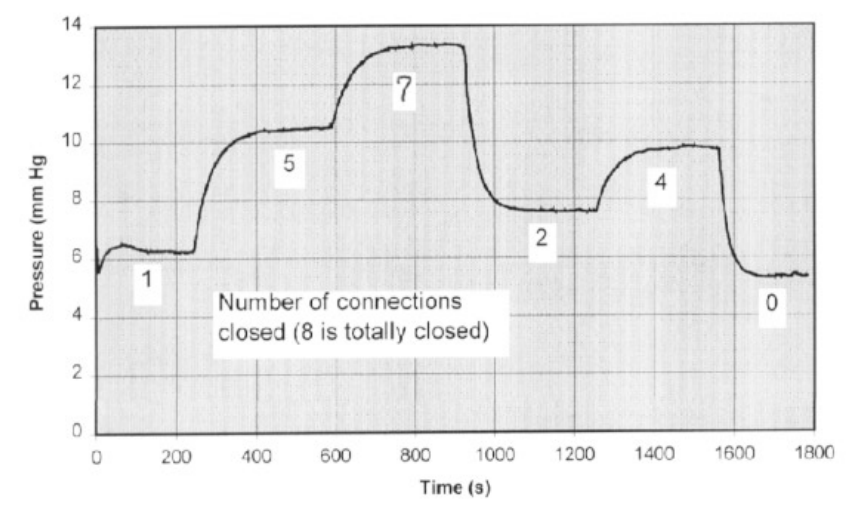

Figure 9. Result of flow-pressure measurement at a flow of $2 \mu \mathrm{l} \mathrm{min}$. During the measurement the slide was moved in the positions indicated in the figure.

position (at constant volume flow), the pressure over the valve rises or decreases accordingly. Measurements were repeated several times with different slide positions and at various flow rates to obtain extensive flow-pressure data for all slide positions.

During the measurements no leakage was observed, even when using relatively large pressures during filling of the device. Also leakage under the slide in the closed position was tested by exerting a pressure of $100 \mathrm{~cm}$ water $(\approx 76 \mathrm{mmHg})$ at the inlet for more than $48 \mathrm{~h}$ and collecting fluid exiting the outlet in a small beaker, which was covered to prevent evaporation of the water. Also in this case no leakage could be observed indicating a good closure of the device at these pressures.

If the manipulation of the slide, monitored through a stereo microscope, was done carefully, the Teflon foil would stretch but not rupture. If rupture occurred it was at the sharp edges of the slide. By performing an isotropic after-etch these edges could be rounded to decrease this effect.

\section{Discussion}

As can be seen in figure 9 the regulator can be used to switch between various pressure differences at a constant flow. This property is used to regulate the eye pressure in 
Valve 3 - Pressure at constant flow

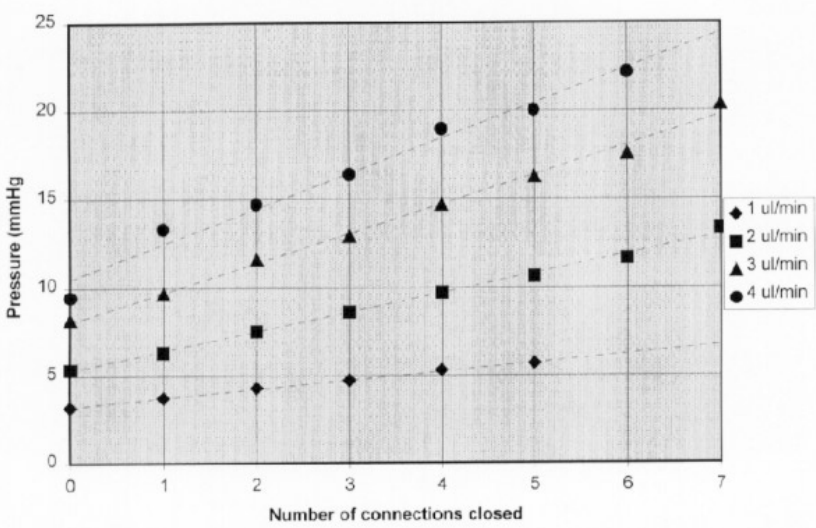

Figure 10. Valve pressure drop as a function of the number of connections closed (eight is totally closed). As expected a linear relation is observed.

glaucoma, as the amount of eye fluid to be transported out of the eye is constant. The settling time that is visible in the results is probably a result of some air that was present at both sides of the pressure sensor, which introduces a capacity parallel to the resistor. The data collected during the measurements at various flow rates were used to establish the pressure difference over the valve at certain flow rates as a function of the number of connections closed. This relation is given in figure 10 for the different flow rates used. The valve was designed to have a linear relation between the number of connections closed and the pressure drop over the valve at a constant flow, or, in other words, the resistance increase was designed to be equal for closure of an additional connection. This linear relation can be observed in figure 10. Furthermore it can be seen that the pressure drop over the valve increases linearly with the flow at a certain slide position (constant resistance value), as could be expected from the relation

$$
p=R \varphi_{v}
$$

\section{Conclusions}

A new valve concept is proposed. Instead of varying the diameter of a channel to alter the resistance of the device, the channel length is manipulated. Various valves and switches have been designed and realized using this concept. A fluid channel switch was realized using a Teflon slide powered by a stepper motor. A glaucoma filter implant valve has been realized in silicon using silicon micromachining techniques. This regulator can increase and decrease the resistance in steps by altering the total channel length using a sliding mechanism. Measurements correspond with theory and show that the regulator concept is suitable for regulating the flow at constant pressure or vice versa. In particular the concept seems to be suitable for regulating the eye pressure in glaucoma.

\section{References}

[1] van der Schoot B H, Verpoorte E M J, Jeanneret S, Manz A and de Rooij N F 1994 Micro Total Analysis Systems ed A van der Berg and P Bergveld (Dordrecht: Kluwer) p 73

[2] Fluitman J H J, van den Berg A and Lammerink T S J 1994 Micro Total Analysis Systems ed A van der Berg and $\mathrm{P}$ Bergveld (Dordrecht: Kluwer) p 73

[3] Lammerink T S J, Spiering V L, Elwenspoek M, Fluitman J H J and van den Berg A 1996 Modular concept for fluid handling systems Proc. Microelectromechanical Systems (MEMS), San Diego 1996 pp 389-94

[4] Breedveld P C, Breunese A P J and Broenink J F et al Modelvorming en simulatie Lecture notes course 'Modelling and Simulation' 1993/1994 (University of Twente, Enschede, the Netherlands)

[5] Shah R K 1978 Laminar Flow Forced Convection in Ducts (New York: Academic)

[6] van Toor M W 1995 Design and realization of a manually adjustable, implantable valve for use in a glaucoma filter implant RTD Thesis MESA Research Institute, University of Twente, The Netherlands

[7] Jansen H V, Gardeniers J G E, Elders J, Tilmans H A C and Elwenspoek M 1994 Applications of fluorocarbon polymers in micromechanics and micromachining Sens. Actuators A 41-42 136-40 